\title{
MULTIPLICATIVE SUBGROUPS OF FINITE INDEX IN A DIVISION RING
}

\author{
GERHARD TURNWALD
}

(Communicated by Maurice Auslander)

\begin{abstract}
If $G$ is a subgroup of finite index $n$ in the multiplicative group of a division ring $F$ then $G-G=F$ or $|F|<(n-1)^{4}+4 n$. For infinite $F$ this is derived from the Hales-Jewett theorem. If $|F|>(n-1)^{2}$ and -1 is a sum of elements of $G$ then every element of $F$ has this property; the bound $(n-1)^{2}$ is optimal for infinitely many $n$.
\end{abstract}

\section{INTRODUCTION}

It is well known that every nonzero element of a finite field $F$ is a sum of two nonzero $n$th powers if $q=|F|$ is sufficiently large. Since $F^{*}$ is cyclic, this is equivalent to the statement that, for every positive integer $n, G+G \supseteq$ $F^{*}$ holds if $G$ is a subgroup of index $n$ of $F^{*}$ provided $q \geq q_{0}(n)$. Leep and Shapiro gave a proof for $n=3$ which also works for infinite fields; they conjectured that $G+G=F$ holds for $n=5$ if $F$ is an infinite field [3]. Recently, Berrizbeitia proved that $G-G=F$ if $\operatorname{char} F=0$ or char $F \geq p_{0}(n)$. $\left(G-G\right.$ means $\left\{g_{1}-g_{2}: g_{1}, g_{2} \in G\right\}$.) Thus, in particular, $G+G=F$ if $n$ is odd and char $F=0$. (Note that $-1=(-1)^{n} \in G$.) The proof in [1] is based on Gallai's theorem (cf. 1.2) which does not give (reasonable) bounds for $p_{0}(n)$. Employing the Hales-Jewett theorem, a modification of Berrizbeitia's proof allows us to prove the following result for infinite $F$.

Theorem 1. Let $F$ be a division ring and $G$ be a subgroup of $F^{*}$ with finite index $n$. If $|F| \geq(n-1)^{4}+4 n$ then $G-G=F$; if, in addition, $n$ is odd then $G+G=F$.

Thus $G-G=F$ holds if $|F| \geq n^{4}$ and $|F|>2$. Choosing $F=\mathbf{F}_{p^{2}}$ and $G=\mathbf{F}_{p}^{*}$ shows that $|F| \geq(n-1)^{2}$ is not sufficient if $n-1$ is a prime. A more elaborate example shows that, for infinitely many $n,|F| \geq(n+1)^{2}$ is not sufficient (see Proposition 1.6).

The notation of Theorem 1 will be kept throughout the paper except in Corollary 1.2. $\mathbf{N}$ denotes the set of positive integers. For every $k \in \mathbf{N}$ we put $G_{k}=\left\{g_{1}+\cdots+g_{k}: g_{1}, \ldots, g_{k} \in G\right\}$ and $S_{k}=G_{1} \cup \cdots \cup G_{k}$. Let $S=\bigcup_{k \geq 1} S_{k}$.

Received by the editors May 26, 1992.

1991 Mathematics Subject Classification. Primary 12E99; Secondary 05D10, 11T99, 12E15.

Key words and phrases. Multiplicative subgroup, field, division ring, Hales-Jewett theorem. 
Theorem 2. If $|F|>(n-1)^{2}$ and $-1 \in S$ then $S=F$.

1 Remark 2.3 shows that the bound for $|F|$ is optimal for infinitely many $n$. The proof is similar to the proof given by Leep and Shapiro for infinite $F$ [3, Lemma 1]. The following theorem refines the results of $\S 2$ in [1].

Theorem 3. (i) If $G \subseteq G-G$ then $S_{k}=G_{k}$ for all $k \in \mathbf{N}$.

(ii) $S_{k} \subseteq S_{k+1}$ for every $k \in \mathbf{N} ; S_{k}=S_{k+1}$ iff $S_{k}=S$.

(iii) $S_{n+1}=S$.

(iv) If $-1 \notin S$ then $n$ is even and $S_{n / 2}=S$.

The examples given in Remark 2.5 show that the bounds in (iii) and (iv) are optimal for infinitely many $n$.

\section{Results CONCERNING $G-G$ AND $G+G$}

1.1. Theorem (Hales-Jewett). For all $m, r \in \mathbf{N}$ there exists $N(m, r) \in \mathbf{N}$ such that, for every $N \in \mathbf{N}$ with $N \geq N(m, r)$, every function $f$ defined on $\{0, \ldots, m\}^{N}$ with at most $r$ values is constant on some line.

(A line is a set of the form $\left\{\left(k_{1}, \ldots, k_{N}\right): k_{j}=k_{j}^{\prime}\right.$ if $j \in J_{0}$ and $k_{j_{1}}=k_{j_{2}}$ if $\left.j_{1}, j_{2} \in J_{1}\right\}$ for suitable disjoint $J_{0}, J_{1}$ with $\{1, \ldots, N\}=J_{0} \cup J_{1}, J_{1} \neq \varnothing$, and suitable $k_{j}^{\prime} \in\{0, \ldots, m\}$ for $\left.j \in J_{0}.\right)$

For a proof we refer to [2]; note that $t$ and 0 have to be interchanged in the definition of $x_{i j}, y_{s i}$ on p. 37 in [2].

1.2. Corollary. Let $S^{\prime}$ be a finite subset of a commutative semigroup $S$. Then for every mapping $g$ from $S$ into some finite set there exist $s \in S$ and $d \in \mathbf{N}$ such that $g$ is constant on $\left\{s+d s^{\prime}: s^{\prime} \in S^{\prime}\right\}$.

Proof. We may assume $S^{\prime}=\left\{s_{0}, \ldots, s_{m}\right\}$ with $m \geq 1$. The assertion follows by applying 1.1 to $f\left(k_{1}, \ldots, k_{N}\right)=g\left(\sum_{j=1}^{N} s_{k_{j}}\right) \quad\left(0 \leq k_{j} \leq m\right)$ for suitably large $N$.

Gallai's theorem is the special case $S=\mathbf{R}^{m}$ (cf. [2, p. 38]) or $S=\mathbf{N}_{0}^{m}$ (as used in [1]). Van der Waerden's theorem on arithmetic progressions is obtained for $S=\mathbf{N}$ or $S=\mathbf{N}_{0}$. Corollary 1.2 is not required in the sequel.

1.3. Proposition. Let $F$ be an infinite division ring and $G$ be a subgroup of $F^{*}$ of finite index $n$. Then for arbitrary $x_{1}, \ldots, x_{m} \in F^{*}$ there exists $c \in F^{*}$ such that $1+c x_{k} \in G$ for $1 \leq k \leq m$.

Proof. For every $N \in \mathbf{N}$ there exist $c_{1}, \ldots, c_{N} \in F$ such that $\sum_{j \in J} c_{j} \neq 0$ for every nonempty $J \subseteq\{1, \ldots, N\}$. (Inductively, $c_{k}$ can be chosen such that $\sum_{j \in J} c_{j} \neq 0$ for all $J \subseteq\{1, \ldots, k\}$.) Now let $N=N(m, n+1)$ (according to Theorem 1.1), set $x_{0}=0$, and set $f\left(k_{1}, \ldots, k_{N}\right)=\left(\sum_{j=1}^{N} c_{j} x_{k_{j}}\right) G$ (where $c G=\{c x: x \in G\})$ for all $k_{j} \in\{0, \ldots, m\}$. By Theorem 1.1 there exist disjoint $J_{0}, J_{1}$ with $\{1, \ldots, N\}=J_{0} \cup J_{1}, J_{1} \neq \varnothing$, and $k_{j}^{\prime} \in\{0, \ldots, m\}$ such that $a G=\left(a+b x_{k}\right) G$ for $1 \leq k \leq m$, where $a=\sum_{j \in J_{0}} c_{j} x_{k_{j}^{\prime}}$ and $b=\sum_{j \in J_{1}} c_{j}$. The assertion holds with $c=a^{-1} b$. (Note that $a \neq 0$ since $b \neq 0$ and $x_{k} \neq 0$.)

\footnotetext{
${ }^{1}$ Note that $-1=p-1 \in G_{p-1} \subseteq S$ if $p=\operatorname{char} F>0$.
} 
1.4. Proof of Theorem 1. If $-1 \notin G$ then $G$ has index 2 in $G\langle-1\rangle$ and hence $n$ is even. Thus it remains to show $G-G=F$. If $F$ is infinite then applying Proposition 1.3 to any left diagonal of $G$ yields a left diagonal $x_{1}, \ldots, x_{n}$ of $G$ such that $1+x_{k} \in G$ (and hence $x_{k} G \subseteq G-G$ ) for $1 \leq k \leq n$; thus $F \subseteq G-G$. Now let $F$ be finite. By Wedderburn's theorem $[4,2.55]$ we have $F=\mathbf{F}_{q}$ for suitable $q$. Thus $F^{*}$ is cyclic and $G=\left\{x^{n}: x \in F^{*}\right\}$. It is well known that the number $N$ of solutions $(x, y) \in F \times F$ of $x^{n}-y^{n}=c$ satisfies $|N-q| \leq(n-1)^{2} \sqrt{q}$ if $c \in F^{*}[4,6.37]$. Let $q=(n-1)^{4}+d$ with $d \geq 4 n$. If $n>1$ then $(n-1)^{2}+(n-1)^{-2}(d-2 n)>\sqrt{q}$ and thus $N \geq q-(n-1)^{2} \sqrt{q}>2 n$. If $n=1$ then $N=q \geq 4$. Since the number of solutions with $x=0$ or $y=0$ is at most $2 n$, this shows that $c \in G-G$.

1.5. Remark. If $n=2$ then $G-G=F$ unless $|F| \in\{3,5\}$ in which case $G-G=F \backslash\{1,-1\}$. If $n=3$ then $G-G=F$ unless $|F| \in\{4,7,13,16\}$. The exceptional cases are $G-G=\{0\}$ for $|F|=4, G-G=\{0,2,-2\}$ for $|F|=7$, and $G-G=F \backslash G$ for $|F| \in\{13,16\}$.

By using Theorem 1 and the fact that $n$ divides $|F|-1$ it only remains to check three cases for $n=2$ and six cases for $n=3$. We omit the details. A self-contained proof of (the first part of) the assertion for $n=3$ can be found in [3].

1.6. Proposition. There are infinitely many $n$ such that $|F|=(n+1)^{2}$ and $G-G \neq F$.

Proof. Let $p>3$ be a prime such that -3 is a square mod $p$. By the quadratic reciprocity law this holds for every prime $p \equiv 1(\bmod 12)$ and by Dirichlet's theorem there exist infinitely many such $p$. Let $F=\mathbf{F}_{p^{2}}$ and $G=\{x \in$ $\left.F: x^{p+1}=1\right\}$; then $G$ has index $n=p-1$ in $F^{*}$. Assume that $-1 \in G-G$, i.e., there exists $x \in F^{*}$ with $x^{p+1}=(x-1)^{p+1}=1$. Taking into account that $(x-1)^{p}=x^{p}-1$ this yields $\left(x^{-1}-1\right)(x-1)=1$. Hence $x^{2}-x+1=0$ which gives $x=(1+a) / 2$, where $a^{2}=-3$. By assumption we have $a \in \mathbf{F}_{p}$; hence $x \in \mathbf{F}_{p}$ and $x^{p-1}=1$. From $x^{p+1}=1$ and $x^{2}-x+1=0$ we thus deduce $x=2$ and $a=3$. Clearly, this is impossible.

1.7. Remark. If $|F|$ is finite then in Theorem 1 one gets $G+G \supseteq F^{*}$. This is proved by an obvious modification of the proof of $G-G=F$. If $G+G \supseteq F^{*}$ then $G+G=F$ holds iff $-1 \in G$, i.e., iff $(-1)^{(|F|-1) / n}=1$.

For infinite $F$ the situation is different since $G=\left\{2^{k} \frac{a}{b}: k \equiv 0\left(\bmod \frac{n}{2}\right)\right.$; $a, b \in \mathbf{N} ; a, b$ odd $\}$ is a subgroup of (even) index $n$ in $\mathbf{Q}^{*}$ and $G+G$ is a proper subset of $\mathbf{Q}^{*}$ (by positivity). Hence for infinite $F$ we cannot conclude $F^{*} \subseteq G+G$. We do have $G \subseteq G+G$, however, since $G \subseteq G-G$ (and hence some element of $G$ belongs to $G+G)$.

1.8. Remark. Let $(*)$ denote the statement $(G \cap \mathbf{Z})-(G \cap \mathbf{Z})=\mathbf{Z}$. The following examples show that $(*)$ holds in several cases but does not hold in general (for $F=\mathbf{Q})$.

(i) Let $p$ be prime. Then $G=\left\{p^{k} \frac{a}{b}: k, a, b \in \mathbf{Z}, a \equiv b \not \equiv 0(\bmod p)\right\}$ is a subgroup of finite index of $\mathbf{Q}^{*}$ (cf. Remark 2.5). Clearly, $x \in G \cap \mathbf{Z}$ implies $x \equiv 0,1(\bmod p)$ and hence $(*)$ does not hold if $p>3$.

(ii) $G=\left\{(-2)^{k} 9^{l} \frac{a}{b}: k, l \in \mathbf{Z} ; a, b \in \mathbf{N}\right.$ with $\left.(a b, 6)=1\right\}$ has index 4 in $\mathbf{Q}^{*}$. Note that $\mathbf{Z} \subseteq\{1,-1,3,-3\} \cdot(G \cap \mathbf{Z})$. Hence $(*)$ holds since $1=5-4$, $3=7-4$, and $4,5,7 \in G \cap \mathbf{Z}$. 
(iii) $G=\left\{\prod_{p \text { prime }} p^{k_{p}}: k_{p} \in \mathbf{Z}, k_{p}=0\right.$ for large $p, \sum k_{p}$ even $\}$ has index 4 in $\mathbf{Q}^{*}$. For every prime $p,\{1,-1, p,-p\}$ is a diagonal of $G$. It is, however, easy to see that there exists no finite set $M \subseteq \mathbf{Z}$ with $\mathbf{Z} \subseteq M \cdot(G \cap \mathbf{Z})$. In order to prove $(*)$ it is sufficent to show that $(G \cap \mathbf{Z})-(G \cap \mathbf{Z})$ contains 1 and all primes $p$. Now note that $1=10-9,2=6-4$, and $2 j-1=j^{2}-(j-1)^{2}$ (for $j>1$ ).

(iv) Choose $m \in \mathbf{N}$ and $c_{p} \in \mathbf{Z}$ (for every prime $p$ ), where $c_{p}=0$ for large $p$. Then $G=\left\{ \pm \prod_{p \text { primes }} p^{k_{p}}: k_{p} \in \mathbf{Z}, k_{p}=0\right.$ for large $p, \sum c_{p} k_{p} \equiv$ $0(\bmod m)\}$ has index $\leq m$. Consider nonnegative integers $l_{p}$ such that $l_{p}=0$ for large $p$. Set $k_{p}=m$ if $c_{p} \neq 0, l_{p}=0$; set $k_{p}=0$ in all other cases. It is then easy to see that $\prod p^{k_{p}}$ and $\prod p^{k_{p}}-\prod p^{l_{p}}$ both belong to $G \cap \mathbf{Z}$ which proves $(*)$ since $-1 \in G \cap \mathbf{Z}$.

\section{Results CONCERNING $G_{k}, S_{k}$, AND $S$}

2.1. Proposition. $S+S \subseteq S$ and $S^{*}=S \backslash\{0\}$ is a group.

Proof. Obviously, $S+S \subseteq S$ and $S \cdot S \subseteq S$. If $x \in F^{*}$ then $x^{m} \in G$ for some $m \in \mathbf{N}$ since otherwise all cosets $x^{k} G(k \in \mathbf{Z})$ are distinct. Thus $x^{-1}=x^{m-1} x^{-m} \in S$ if $x \in S^{*}$.

2.2. Proof of Theorem 2. Let $-1 \in S$ and assume that there exists $x \in F \backslash S$. The cosets $(a+x) G$ with $a \in G \cup\{0\} \subseteq S$ are distinct since $a+x=\left(a_{1}+x\right) a_{2}$ with $a, a_{1}, a_{2} \in S$ yields $x\left(a_{2}-1\right)=a-a_{1} a_{2} \in S$ and hence (by Proposition 2.1) $a_{2}-1=0, a=a_{1}$. Moreover, $a+x \neq 0$ and $(a+x) G \neq G$. Hence $|G|+2 \leq n$ and $|F|=n|G|+1 \leq(n-1)^{2}$.

2.3. Remark. Let $F=\mathbf{F}_{q^{2}}$ and $G=\mathbf{F}_{q}^{*}$. Then $n=q+1$ and $-1 \in S \subseteq \mathbf{F}_{q} \neq$ $F$. Since $|F|=(n-1)^{2}$, this shows that the bound in Theorem 2 is optimal for infinitely many $n$.

2.4. Proof of Theorem 3. (i) Some element of $G$ belongs to $G+G$ and thus $G \subseteq G+G$. Inductively, $S_{k} \subseteq G_{k}$ for all $k$ and hence $S_{k}=G_{k}$.

(ii) This is evident from the definitions.

(iii) For every $k \in \mathbf{N}, S_{k}$ is a union of cosets of $G$ possibly together with $\{0\}$. Thus the assertion follows from (ii).

(iv) $n$ is even since $-1 \notin G$ (cf. Proof 1.4). We have $0 \notin S$ since otherwise $0 \in G_{k}$ for some $k \geq 2$ and hence $-1 \in G_{k-1} \subseteq S$. Thus (by 2.1) $S$ is a subgroup of $F^{*}$. Since $G \leq S \neq F^{*}$, we obtain $(S: G) \leq n / 2$ and thus (ii) yields $S_{n / 2}=S$ (since each $S_{k}$ is a union of cosets of $G$ ).

2.5. Remark. It is easy to see that $G \subseteq G-G$ is equivalent to $G \subseteq G+G$. According to Theorem 1, the hypothesis $G \subseteq G-G$ may be omitted in (i) if $|F| \geq(n-1)^{4}+4 n$. Choosing $G=\{1\}$ shows that some additional assumption is required in general.

Now let $F=\mathbf{Q}$ and define $G$ as in Remark 1.8(i). Note that $G$ has index $p-1$ and $1, \ldots, p-1$ is a diagonal. If $1 \leq k<p$ then, putting $l=p-1-k$ and $G_{0}=\{0\}$, we have $k=(1-l p)+k-1+l p \in G+G_{k-1}+G_{l}=G_{p-1}$. Hence $F^{*} \subseteq G_{p-1}$ and $S=F$. It is easy to see that $0 \notin G_{p-1}$ and thus, since $S_{k}=G_{k}$ for all $k, S_{p-1} \neq S$. Consequently, the index $n+1$ in (iii) is optimal if $n+1$ is a prime (cf. $[1, \S 3]$ ). Since $G$ contains negative elements (e.g., $1-p$ ), the subgroup $G_{+}$of positive elements of $G$ has index 2 in $G$ 
and hence $\left(F^{*}: G_{+}\right)=2(p-1)$. We have $p-1 \notin S_{p-2}$ since otherwise $0=(p-1)+(1-p) \in S_{p-1}=G_{p-1}$. Since every positive integer is a sum of elements of any given subgroup, this shows that the index $\frac{n}{2}$ in (iv) is optimal if $n=2(p-1)$ for some prime $p$.

2.6. Remark. In Proposition 2.1(b) of [1] it is stated that $-1 \in S$ implies $S_{n+1}=F$. (The notation $k \times G, P_{k}, P$ in [1] corresponds to $G_{k}, S_{k}, S$ used in this paper.) This is correct if $F$ is infinite (cf. Theorem 2) but may fail for finite fields (cf. Remark 2.3). (In [1] a result is quoted from [3] without the hypothesis on $|F|$ made there.) Theorem 3(iv) improves the second part of Proposition 2.1(b) of [1]; thus the title of $\S 3$ in [1] is misleading.

2.7. Remark. Let $k>1$. It is easy to see that $0 \in G_{k}$ holds iff $-1 \in G_{k-1}$. If $-1 \in G_{k-1}$ and $G-G=F$ then $F \subseteq G+G_{k-1}=G_{k}$ (cf. [1, 1.2]). Thus the following three statements are equivalent if $G-G=F: G_{k}=F, 0 \in G_{k}$, $-1 \in G_{k-1}$; moreover, $G_{k}=S_{k}$ (by Theorem 3(i)).

\section{NOTE ADDED IN PROOF}

For infinite $F$ Theorem 1 is a special case of the results in a recently published paper by V. Bergelson and D. B. Shapiro (Multiplicative subgroups of finite index in a ring, Proc. Amer. Math. Soc. 116 (1992), 885-896). Their proof is based on the amenability of abelian groups and a simple version of Ramsey's Theorem.

\section{REFERENCES}

1. P. Berrizbeitia, Additive properties of multiplicative subgroups of finite index in fields, Proc. Amer. Math. Soc. 112 (1991), 365-369.

2. R. L. Graham, B. L. Rothschild, and J. H. Spencer, Ramsey theory, Wiley, New York, 1980.

3. D. B. Leep and D. B. Shapiro, Multiplicative subgroups of index three in a field, Proc. Amer. Math. Soc. 105 (1989), 802-807.

4. R. Lidl and H. Niederreiter, Finite fields, Addison-Wesley, Reading, MA, 1983.

Mathematisches Institut, Universität TÜbingen, Auf Der Morgenstelle 10, D-72076 Tübingen, Federal Republic of Germany

E-mail address: turnwald@mailserv.zdv. uni-tuebingen.de 